\title{
On Some Properties of Positive and Strongly Positive Arithmetical Sets
}

\author{
Seda N. Manukian \\ Institute for Informatics and Automation Problems of NAS RA \\ e-mail: zaslav@ipia.sci.am
}

\begin{abstract}
The notions of positive and strongly positive arithmetical sets are defined as in [1]-[4] (see, for example, [2], p. 33). It is proved (Theorem 1) that any arithmetical set is positive if and only if it can be defined by an arithmetical formula containing only logical operations $\exists, \&, \vee$ and the elementary subformulas having the forms $x=0$ or $x=y+1$, where $x$ and $y$ are variables.

Corollary: the logical description of the class of positive sets is obtained from the logical description of the class of strongly positive sets replacing the list of operations $\&, \mathrm{~V}$ by the list $\exists, \&, \mathrm{~V}$. It is proved (Theorem 2 ) that for any one-dimensional recursively enumerable set $M$ there exists 6-dimensional strongly positive set $H$ such that $x \in M$ holds if and only if $\left(1,2^{x}, 0,0,1,0\right) \in H^{+}$, where $H^{+}$is the transitive closure of $H$.

Keywords: Positive set, Strongly positive set, Transitive closure, Signature.
\end{abstract}

\section{Introduction}

In this article some simplified forms are considered for the representation of any positive arithmetical set (see below, Theorem 1). The method used in [2] for the construction of a creative set on the base of the transitive closure of some strongly positive set is generalized below, and it is shown that similar method gives the possibility for obtaining any one-dimensional recursively enumerable set on the base of the transitive closure of some strongly positive set (Theorem 2).

The formulations of Theorem 1 and its corollary are given (without proof) in [4]. 


\section{Main Notions and Definitions}

By $N$ we denote the set of all non-negative integers $\{0,1,2, \ldots\}$. By $N^{n}$, where $n \geq 1$, we denote the set of all $n$-tuples $\left(x_{1}, x_{2}, \ldots, x_{n}\right)$, where $x_{i} \in N$ for $1 \leq i \leq n$. The $\underline{n \text {-dimensional arithmetical }}$ set, where $n \geq 1$ is defined as any subset of $N^{n}$. The $\underline{n \text {-dimensional arithmetical predicate is }}$ defined in the usual way as a predicate, which is true on some set $A \subseteq N^{n}$ and false on the set $N^{n} \mid A$ (cf. [5]-[6]).

The notions of general recursive function, partial recursive function and all the notions connected with them are defined as in ([5]-[6]).

Signature is defined as usual, as any set of predicate symbols, functional symbols and symbols of constants. The notion of arithmetical formula in a given signature is defined in the usual way (see, for example, [2]); we will consider predicate formulas in the signature $(0,=, S)$, where $S(x)=x+1$ for $x \in N$. The expressions $S(S(\ldots S(x) \ldots))$ and $S(S(\ldots S(0) \ldots))$, where the symbol $S$ is repeated $n$ times, we will shortly denote as $S^{n}(x)$ and $S^{n}(0)$ (cf.[2]).

The deductive arithmetical system in the signature $(0,=, S)$ is defined as in [7]; it is proved in [7] that this system is complete.

All auxiliary notions connected with the notions mentioned above are defined as in [2].

For the convenience of the reader, let us recall the definitions of positive and strongly arithmetical set.

An arithmetical set is said to be positive if it can be defined by an arithmetical formula in the signature $(0,=, S)$ such that it contains no other symbols of logical operations besides $\exists, \&, \mathrm{~V}, \neg$ and has the following property: all the symbols $\neg$ relate to the elementary subformulas containing no more than one variable. An arithmetical set is said to be strongly positive if it can be defined by an arithmetical formula in the signature $(0,=, S)$ such that it contains no other logical operations besides $\&$ and $\mathrm{V}$ relating to the formulas having one of the forms $x=a, x=y, x=S(y), \neg(x=$ 0 ), where $x$ and $y$ are variables and $a$ is a constant.

The transitive closure of a set having an even dimension is defined in the usual way (see, for example, [2], p.34).

\section{Main Theorems}

Theorem 1: Any arithmetical set is positive if and only if it can be defined by an arithmetical formula, which contains only logical operations $\exists, \&, \vee$ and such that all elementary subformulas in it have the form $x=0$ or $x=S(y)$.

Proof. Let $F$ be any arithmetical formula defining a positive arithmetical set. Any subformula of $F$ containing the negation $\neg$ may be reduced to the form $\neg\left(x=S^{n}(0)\right)$ or $\quad \neg\left(S^{n}(x)=0\right)$. However, the formula $\neg(x=0)$ is equivalent to $\exists z(x=S(z))$ and the formula $\neg\left(x=S^{n}(0)\right)$ when $n>0$ is equivalent to the formula $(x=0) \vee(x=S(0)) \vee \ldots \vee\left(x=S^{n-1}(0)\right) \vee \exists z(x=$ $\left.S^{n+1}(z)\right)$.

The formula $\neg\left(S^{n}(x)=0\right)$ is equivalent to $\exists z(x=S(z))$ when $n=0$ and is equivalent to $x=x$ when $n>0$. So, in all cases there exists a formula $F_{1}$, which is equivalent to $F$ and does not contain the symbol $\neg$.

Any elementary subformula of $F_{1}$ may be reduced to the form $\left(x=S^{n}(y)\right)$, where $x$ and $y$ are variables (or, may be, constants). But any formula having the form $\left(x=S^{n+1}(y)\right)$ may be transformed into $\exists z\left(x=S^{n}(z) \&(z=S(y))\right)$. Using a similar transformation, we may reduce the 
formula $x=S^{n}(y)$ to the form in which all the elementary subformulas have the form $u=0$ or $v=S(w)$, where $u, v, w$ are variables. This completes the proof.

Corollary 1: The definition of a positive set is obtained when we replace the list of operations $\&, \vee$ with the list $\exists, \&, \vee$ in the definition of a strongly positive set. The proof is easily obtained from the considerations given in the proof of Theorem 1.

Theorem 2: For any one-dimensional recursively enumerable set $M$ there exists a 6-dimensional strongly positive set $H$ such that $x \in M$ takes place if and only if $\left(1,2^{x}, 0,0,1,0\right) \in H^{+}$, where $H^{+}$is the transitive closure of $H$.

Proof. Let $M$ be any one-dimensional recursively enumerable set. Clearly, there exists a partial recursive function $f(x)$ such that $f(x)=0$ when $x \in M$ and $f(x)$ is undefined when $x \notin M$. We will use the notions of $\Omega$-algorithm (see [2], pp. 34-35) and $\Gamma_{2}$-algorithm (see [2], pp. 35-36) defined in [2]. Below we will use the notion of $\Gamma_{n}$-algorithm only in the case when $n=2$. Using Theorem 3 in [2] (see [2], p. 35 and also [6], pp. 312-315) and [8], we conclude that there exists an $\Omega$-algorithm $\theta$ such that it transforms the state $\left(1,2^{2^{x}}\right)$ into the state $(0,2)$, when $x \in M$, and is not applicable to the state $\left(1,2^{2^{x}}\right)$ when $x \notin M$. Now, using Lemma 3.1, Lemma 3.2 in [2] (see [2], pp. 36-37) we obtain that there exists a $\Gamma_{2}$-algorithm $\psi$ such that it transforms the state $\left(1,2^{x}, 0\right)$ into the state $(0,1,0)$ when $x \in M$ and is not applicable to the state $\left(1,2^{x}, 0\right)$ when $x \notin$ $M$. Let us consider the "step-describing set" (shortly, "SD-set") $H$ for the $\Gamma_{2}$-algorithm $\psi$ (the notion of SD-set for $\Gamma_{2}$-algorithm is given in [2], p. 38). As it is proved in [2], the set $H$ is strongly positive (the proof is actually given in [2], pp. 37-38; see also [2], Lemma 3.3, p. 39). Now, if we denote the transitive closure of $H$ by $H^{+}$, then it is easily seen that $x \in M$ takes place if and only if $\left(1,2^{x}, 0,0,1,0\right) \in H^{+}$. This completes the proof.

\section{References}

[1] S. N. Manukian, "On an algebraic classification of multidimensional recursively enumerable sets expressible in formal arithmetical systems", Transactions of the IIAP of NAS RA, Mathematical Problems of Computer Science, vol. 41, pp. 103-113, 2014.

[2] S. N. Manukian, “On strongly positive multidimensional arithmetical sets", Transactions of the IIAP of NAS RA, Mathematical Problems of Computer Science, vol. 43, pp. 32-41, 2015.

[3] S. N. Manukian, "On transitive closures of two-dimensional strongly positive arithmetical sets", Transactions of the IIAP of NAS RA, Mathematical Problems of Computer Science, vol. 45, pp. 67-76, 2016.

[4] S. N. Manukian, "On the structure of positive and strongly positive arithmetical sets", Proceedings of the International Conference and Information Technologies, CSIT-17, Yerevan, Armenia, p. 33, 2017.

[5] S. C. Kleene, Introduction to Metamathematics, D. van Nostrand comp., Inc. New YorkToronto, 1952.

[6] A. I. Malcev, Algorithms and Recursive Functions, $2^{\text {nd }}$ edition (in Russian), M., "Nauka”, 1986.

[7] H. B. Enderton, A Mathematical Introduction to Logic, $2^{\text {nd }}$ edition, San Diego, Harcourt, Academic Press, 2001.

[8] M. L. Minsky, "Recursive Unsolvability of Post's Problem of "Tag and Other Topics in Theory of Turing Machines”, Ann. Math., vol. 74, pp. 437-455, 1961. 
Submitted 20.07.2018, accepted 02.12.2018.

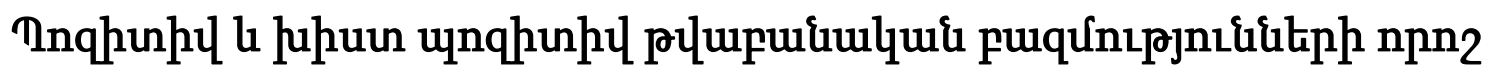 huunlnıpjnzqutph ưupq
}

\author{
U. Uuifunıljuif
}

\section{Uưhnนhnıर}

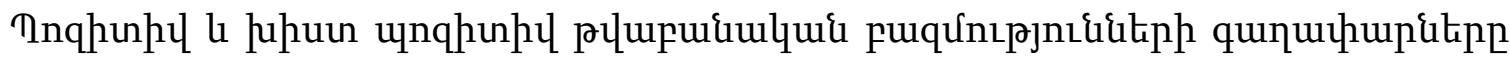

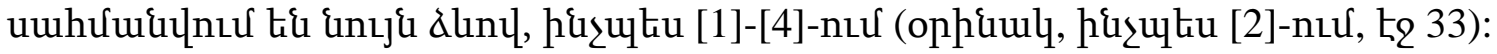

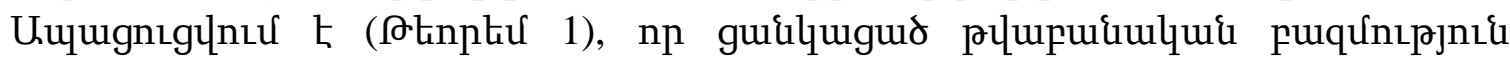

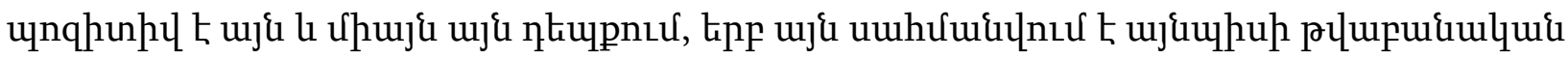

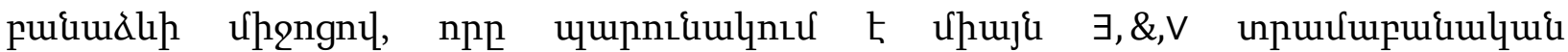

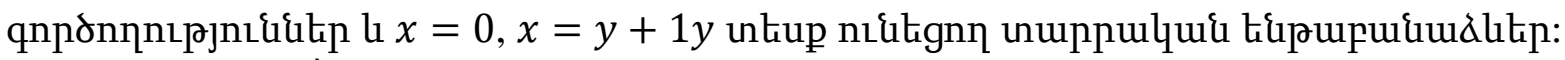

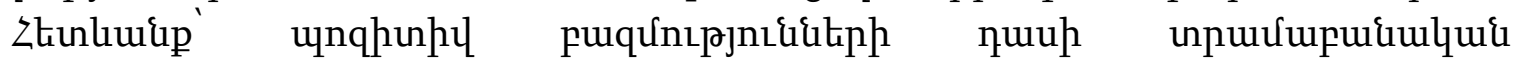

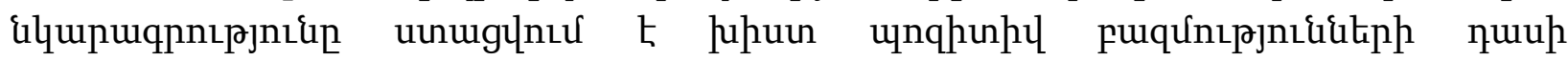

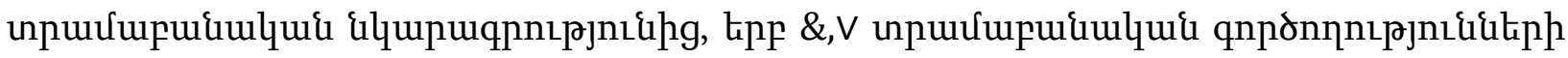

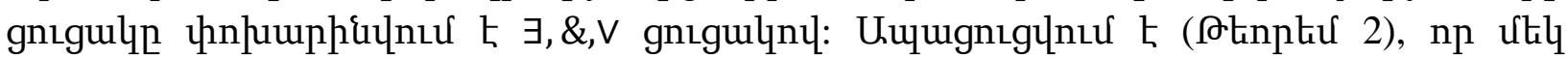

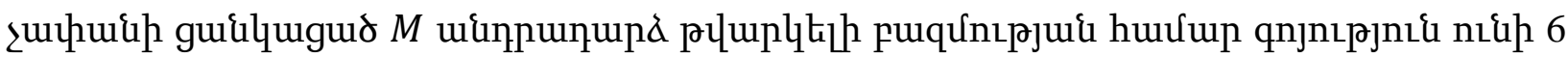

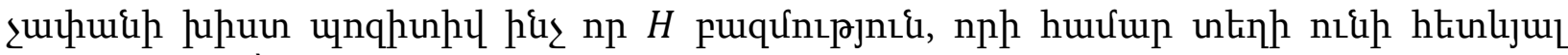

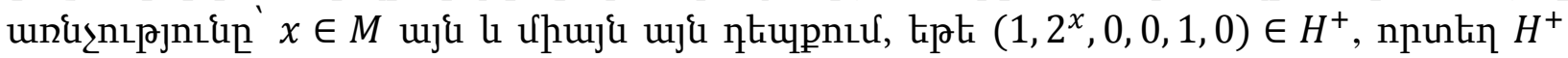

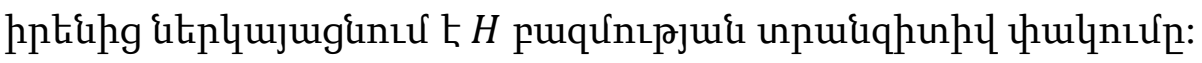

\section{О некоторых свойствах позитивных и строго позитивных арифметических множеств}

\author{
С. Манукян
}

\begin{abstract}
Аннотация
Определения понятий позитивного и строго позитивного арифметического множества даются так же как в [1]-[4] (см., например, [2], стр.33). Доказывается (Теорема 1), что любое арифметическое множество позитивно в том, и только в том случае, когда оно задается арифметической формулой, которая содержит только логические операции $\exists, \&, \mathrm{~V}$ и только элементарные подформулы вида $x=0, x=y+1$.

Следствие: Логическое описание класса позитивных арифметических формул получается из логического описания класса строго позитивных арифметических формул посредством замены списка логических операций \&,V списком $\exists, \&, \mathrm{~V}$. Доказывается (Теорема 2), что для любого одномерного рекурсивно перечислимого множества $M$ существует строго позитивное множество $H$ размерности 6 , такое, что $x \in M$ имеет место в том и только в том случае, когда $\left(1,2^{x}, 0,0,1,0\right) \in H^{+}$, где $H^{+}$есть транзитивное замыкание множества $H$.
\end{abstract}

